\title{
Il dialogo intertestuale di Bufalino con Ripellino: un primo sondaggio su L'amaro miele
}

Le dialogue intertextuel de Bufalino avec Ripellino : les premiers résultats d'une recherche sur L'amaro miele

The Intertextual Dialogue between Bufalino and Ripellino: A Preliminary

Research on L'amaro miele

\section{Giuseppe Traina}

\section{(2) OpenEdition}

Journals

Edizione digitale

URL: http://journals.openedition.org/cei/6997

DOI: $10.4000 /$ cei.6997

ISSN: 2260-779X

Editore

UGA Éditions/Université Grenoble Alpes

Edizione cartacea

ISBN: 978-2-37747-173-7

ISSN: 1770-9571

\section{Notizia bibliografica digitale}

Giuseppe Traina, « Il dialogo intertestuale di Bufalino con Ripellino: un primo sondaggio su

L'amaro miele », Cahiers d'études italiennes [Online], 30 | 2020, Messo online il 01 mars 2020, consultato il 06 mars 2020. URL : http://journals.openedition.org/cei/6997; DOI : https://doi.org/10.4000/cei. 6997

Questo documento è stato generato automaticamente il 6 marzo 2020

(c) ELLUG 


\title{
Il dialogo intertestuale di Bufalino con Ripellino: un primo sondaggio su L'amaro miele
}

\author{
Le dialogue intertextuel de Bufalino avec Ripellino : les premiers résultats d'une \\ recherche sur L'amaro miele \\ The Intertextual Dialogue between Bufalino and Ripellino: A Preliminary \\ Research on L'amaro miele
}

\section{Giuseppe Traina}

1 «Amici che avrei voluto avere: Théo van Gogh, il dottor Čechov, Angelo Maria Ripellino» ${ }^{1}$. Qualunque discorso sul dialogo intertestuale tra Bufalino e Ripellino deve partire da questo aforisma: dalla definizione di 'amico' e dall'accostamento a Théo van Gogh e a Čechov.

Riflettiamo: 'amico' è molto più che 'maestro', 'sodale' o qualsiasi altro epiteto che ci potremmo aspettare in una dichiarazione di vicinanza intellettuale ad autori o personaggi del passato. Indica una vicinanza innanzitutto affettiva, e poi anche di natura intellettuale ${ }^{2}$. Si noti, peraltro, la sequenza dei tre nomi, collocati, a mio parere, in climax: Théo van Gogh è un fratello che fa da sostegno (anche economico) al fratello geniale e malato (si ricordi che Bufalino era figlio unico e si ammalò di tubercolosi); «il dottor Čechov» è, insieme, un confratello di sventura (anch'egli tisico), un collega scrittore (ma dallo stile alquanto diverso) e un possibile sostegno in termini terapeutici, visto che era medico; Ripellino è, come Čechov, un collega di scrittura e un confratello di sventura (la tubercolosi non risparmiò nemmeno lui) ma è, soprattutto, un poeta che, sia pure sotto il velo delle metafore liriche, ha parlato in un libro della sua esperienza in sanatorio, anch'egli - come Bufalino - adoperando uno stile ipermetaforico, caratterizzato da un lessico rutilante, ricchissimo, fedele tanto alla variatio quanto a ben precisi stilemi: Lafortezza d'Alvernia e altre poesie e Diceria dell'untore $^{3}$ stanno sui nostri scaffali a dimostrarlo. 
3 Ripellino confrère ideale, dunque, ancor più di Čechov. Ed è questo il motivo per cui non avrebbe senso impostare il nostro discorso di natura intertestuale su una presunta 'influenza' di Ripellino su Bufalino, e tantomeno su un'angoscia bloomiana. L'esordio tardivo di Bufalino come romanziere (risalente al 1981, cioè tre anni dopo la morte di Ripellino) non può implicare che sia quest'ultimo ad avere influito marcatamente sullo stile di Bufalino. Direi, piuttosto, che due percorsi letterari e stilistici nati parallelamente sono confluiti in una piena consonanza di vedute e di toni, in una comune visione della letteratura e dell'arte come 'meraviglioso' 'balsamo e contravveleno' ai drammi della vita e, anche, procedimento affine alla rêverie poetica che Bachelard ci ha insegnato a riconoscere.

Va, innanzitutto, considerato il fatto che i due autori sono quasi coetanei: anzi, per amor di precisione, Bufalino era nato nel 1920, cioè tre anni prima dell'altro. Lo scrittore di Comiso, che si ammala di tubercolosi nel $1944 \mathrm{e} \mathrm{nel} \mathrm{'47} \mathrm{è} \mathrm{dichiarato} \mathrm{guarito,}$ comincia a scrivere Diceria dell'untore, ancora in forma di abbozzi e frammenti, tra la fine degli anni Quaranta e i primi Cinquanta, poi ne interrompe la stesura per riprenderla e infine completarla nel ' 71 . Ma non era uno scrittore del tutto inedito, se consideriamo le prose pubblicate sulle riviste «L'Uomo» e «Democrazia», proprio negli anni della malattia e tramite l'amico Angelo Romanò: testi che già dimostrano la qualità innanzitutto lirica della sua prosa, non a caso poi parzialmente confluiti nel romanzo. Dunque, Bufalino, dal fondo della sua provincia siciliana, pubblica poche ma già notevoli prose quando Ripellino, anch'egli di origine siciliana ma presto inseritosi nell'ambiente culturale romano, aveva già da qualche anno cominciato a collaborare regolarmente con riviste come «Meridiano di Roma», «Roma fascista» o «Maestrale», che però mi sento di escludere siano state accessibili a Bufalino. Il quale si accosta alla prosa critica ripelliniana verosimilmente quando ne legge alcune traduzioni, le antologie Poesia russa del Novecento e Nuovi poeti sovietici e i principali libri di saggistica ${ }^{4}$, per poi approdare alla produzione poetica di Ripellino, probabilmente ignorandone il primo lemma, Non un giorno ma adess $0^{5}$, che ebbe invero minima circolazione, ma leggendo i successivi, presenti presso la Biblioteca Comunale di Comiso; e anche presso la sua biblioteca privata, ma solo nel significativo caso di La fortezza d'Alvernia e altre poesie. Come s'è già ricordato, infatti, nella prima ed eponima sezione di questo libro Ripellino raccolse le liriche composte nel biennio 1965-1966, gli anni della salvifica degenza nel sanatorio di Dobřís (la fortezza d'Alvernia, appunto), successiva a una storia di malattia, provvisorie guarigioni e gravi ricadute che era iniziata nei primi anni Quaranta ${ }^{6}$.

5 Insomma, se è vero che Bufalino trovò in tutti i libri (sia lirici che saggistici) di Ripellino una piena consonanza con l'idea, anche sua, di «letteratura come itinerario nel meraviglioso" ${ }^{7}$, anche ragioni di ordine cronologico dimostrano che si può escludere un'influenza intertestuale diretta di Ripellino su Bufalino: sui suoi testi narrativi, poetici, saggistici. In tal senso, appare esagerata la pur meritoria ipotesi di Salvatore Scalia, che ritrovava in Diceria dell'untore «stilemi che dimostrano una inconfondibile ascendenza ripelliniana, oltre che una notevole consonanza sentimentale» ${ }^{8}$.

6 Ma, appunto, meglio parlare di consonanze, che sono tante: nella costruzione del discorso, strutturalmente fondato sulla 'retorica della citazione' e dell'allusione colta (agli spazi della letteratura ma anche dell'arte, del cinema, della musica, del teatro), nell'impianto metaforico del dettato, nella strategia sintattica, nelle predilezioni lessicali, nell'atteggiamento complessivo di fronte alla pagina bianca, che per entrambi 
si configura come lo spazio adatto ad esprimere i tormenti della vita e ibalsami dell'arte, lo strazio e il diletto, il falsetto e il belcanto, il 'trucco' e l'anima'. Riprendo, qui in fondo, un'allusione al titolo di uno dei più bei libri di Ripellino', già adoperata da Nunzio Zago per soffermarsi sul fatto che Ripellino fu «sotto molti riguardi, tematici e formali, tranne che per la vicinanza alla cosiddetta Neoavanguardia (anch'essa, invero, nello stesso Ripellino, più apparente che reale), prossimo ai gusti e alla sensibilità dell'autore comisano» ${ }^{10}$.

7 Per quanto, dunque, impostata nei termini della 'prossimità' e non dell'influenza, un'analisi intertestuale completa andrebbe condotta, e su tutto il corpus dell'opera bufaliniana. Lavoro di gran mole, che rimando ad altra occasione, limitandomi, in questa sede, a un primo sommario regesto, riferito soltanto alla raccolta di poesie di Bufalino L'amaro miele ${ }^{11}$.

Un primo dato che emerge dal confronto tra L'amaro miele e il corpus poetico ripelliniano è di carattere metrico. Agisce in entrambi, e forse più in Bufalino, un forte interesse per il recupero di forme metriche tradizionali, sempre attraversato dal novecentesco senso di libertà che si prova nel trasgredirle: in Bufalino, per esempio, troviamo diversi sonetti regolari oppure un sonetto minore (in settenari: $\mathrm{Su}$ un calendario nuovo, p. 734). Entrambi sembrano però trovare una conciliazione tra queste due spinte in una certa predilezione per la composizione in quartine, più regolarmente assonanzate che rimate: Ripellino soprattutto in Autunnale barocco, la sua ultima raccolta poetica pubblicata in vita $^{12}$, con la preferenza a contenerne la lunghezza fra le due e le quattro quartine; Bufalino diverse volte nell'Amaro miele, anche con soluzioni prolungate che arrivano alle sette quartine di Aegri ephemeris (p.702), in rima o assonanza/consonanza incrociata, e addirittura alle dodici quartine di Didascalie per una visita medica (pp. 703-704), in rima o assonanza alternata.

Dal punto di vista sintattico, non solo il Bufalino prosatore ma anche il poeta dimostra una certa propensione a usare una delle mosse più tipiche dello stile di Ripellino, sia in prosa che in versi, vale a dire l'elencazione, non di rado caotica, talvolta in climax; basti, come minima esemplificazione, la conclusione della quinta ${ }^{13}$ lirica de Lafortezza d'Alvernia: «Amaro è il vedersi in sfacelo nel gorgo / d'un perfido specchio spumoso, l'accorgersi / che il mondo è degli altri, e che gli altri / sono implacabili verso i mollicci, / gli storpi, gli stolidi, i cionchi, i dannati» ${ }^{14}$. Ma si può dire che quasi non vi sia lirica o prosa di Ripellino priva di elencazioni. Reco qualche esempio dall'Amaro miele, riferendomi soltanto a elencazioni che abbiano anche una consonanza di natura tematica con il mondo fantasmagorico di Ripellino:

- «coscritti balbuzienti, spretati dagli occhi miopi, / guitti fischiati, collegiali alla gogna, / re in esilio invecchiati a un tavolo di caffè» (Dedica, dopo molti anni, p. 695, vv. 4-6; lessemi come 'guitti' e 'gogna' o il motivo del re in esilio sono davvero tipicamente ripelliniani);

- «A noi anche Tu devi una donna / che ci schiodi e ci lavi, / un fantaccino cieco che ci vegli, / una resurrezione» (Altri versi scritti sul muro, p. 718, vv. 8-11; in questo caso emerge il grande tema metafisico, di una metafisica esibita e negata - esibita per essere negata -, tipica dei due scrittori);

- «ogni notte più esangui, più scontrosi, più morti» (Agli amici morti 1, p. 727, v. 12);

- «Sulla usata scacchiera / enumeriamo i loschi personaggi, / gualdrappe a lutto, rocche senza tromba, / logori lindi scheletri di bosso, / unghia contr'unghia di sterile luce,» (Allegoria, p. 757, vv. 1-5: dove il gusto dell'enumerazione viene esibito en abyme); 
- «Le mie ragioni, amici: / la metrica e il dolore, l'ordine e la follia, / spazio e mura che cerco tentoni, / gogne guardinghe del cuore...» (Suasoria, p. 766, vv. 1-4; è un tipico esempio di autocommento, prediletto da entrambi i nostri scrittori);

- «O vita, complotto di grandi, / improbo gesto, parola non detta, / nuvola bassa che non fa diluvio» (Lamento del facile cuore, p. 763, vv. 6-8: ipotizzo qui un'eco ripelliniana esplicita, delle sue Notizie dal diluvio ${ }^{15}$;

- «Ci derisero i topi di granaio, / le lascive fameliche colombe, / venne a fiutarci un angelo la mano» (Lamento del facile cuore, p. 763, vv. 10-12: nella stessa poesia, che ha in toto cadenze prettamente ripelliniane, si può addirittura pensare a un esplicito omaggio onomastico, che si somma alla presenza di animali con valore simbolico, anch'essa tipica della poesia di Ripellino).

10 Altra mossa tipicamente ripelliniana è la ripetizione ravvicinata, nello stesso verso o in versi contigui, a volte in anafora, che assume sempre valore enfatico in contesti tendenzialmente drammatici, se non melodrammatici; trascrivo, come esempio ripelliniano, la strofa conclusiva di Notizie dal diluvio - I. 31:

Chi agisce dietro le quinte? Chi manovra le catastrofi?

Chi corrode le corde? Chi custodisce il bestiame?

Chi ci sbalestra nel fango? Chi si diverte a inventare

il cubismo dei crolli e disastri? Da secoli e secoli

sempre lui, sempre lui, sempre lui,

calmo, inesausto, ligio agli orari, inappuntabile ${ }^{16}$.

11 Le diverse figure di ripetizione danno luogo, nella prosa bufaliniana, a splendidi effetti di saporita retorica. Ma anche nell'Amaro miele, magari in forma e quantità più misurata, non mancano esempi interessanti:

- «Ma è sempre un altro, sempre un altro» (Preghiera di mezzogiorno, p. 701, v. 5);

- «Datemi un male senza libri, / datemi un pianto senza specchi» (ibid., vv. 9-10);

- «pendere così freddi, così nudi» (Altri versi scritti sul muro, p. 718, v. 5);

- «Ma tu, cuore, che pensi, che dici» (Esercizio con sentimento, p. 739, v. 9);

- «un'aspide prava, un'aspide storta» (Nascita del peccato, p. 752, v. 5);

- «un'altra attesa, un'altra fuga, un'altra tana» (La sosta, p. 754, v. 11);

- «un altro anno, un'altra storia si consuma» (A Sesta Ronzon, dovunque si trovi, p. 755, v. 11).

Un'altra marca, forse la più tipica, che denota la sintassi ripelliniana è l'abbondanza di forme vocative, che in poesia caratterizzano i testi come soluzioni, spesso audaci, di compromesso tra l'istanza lirico-monologante del discorso (talvolta addirittura sentimentalmente effusivo) e l'apertura, sofferta, a un mondo visto come contrapposto al solipsismo autodifensivo dell'io lirico. Un solo esempio, ad apertura casuale di libro: «Non sono, signori, un Mascagni che si inchini a ringraziare. / Eppure grazie, Kakadù. Grazie, Columba gestuosa / e voi tutti, abitanti della fortezza d'Alvernia» ${ }^{17}$. Ma se questo dato, in Ripellino, si risolve soprattutto in mascheramento di un effettuale e sincero slancio relazionale ${ }^{18}$, in teatrale esibizione di dialogati fittizi, in Bufalino appare quasi sempre come mero espediente retorico, somma di parole rivolte a interlocutori astratti o metaforici, con sovrabbondanza di situazioni e lessico di marca metafisica. Le occorrenze non mancano:

- «Datemi un male senza libri, / datemi un pianto senza specchi» (Preghiera di mezzogiorno, p. 701, vv. 9-10);

- «Dio mio, l'inverno s'avvicina» (Aegri ephemeris, p. 702, v.2). Ma nella stessa lirica - ennesimo dialogo metafisico con un dio poco credibile - troviamo altre forme vocative anche più ripelliniane di questa. Per esempio: «non dirmi che non t'importa» (v. 6), «ma 
chiudimi gli occhi sconfitti / con un lembo del tuo lenzuolo» (vv. 9-10), «o vattene, lasciami solo / nella mia tetra garitta» (vv. 11-12);

- «Venite, venite, veroniche, / con l'acqua, le spugne e le lane» (Didascalie per una visita medica, p. 704, vv. 29-30; e qualche verso dopo: «E tu pungimi, ape regina», v. 33);

- «scoppiami, cuore, che dentro mi bruci; / balzate, piaghe, sulle mie palme felici!» (Altri versi scritti sul muro, p. 718, vv. 8-9);

- «O compagni traditi, / a piedi nudi venitemi dietro, / lapidatemi da lontano» (Lamento del viaggiatore, p. 738, vv. 11-13);

- «Portatemi dunque sulla collina, / ho tante storie da raccontare» (Ritorno sulla collina, p. 740, vv. 9-10);

- «e tu presto ricoprimi / del tuo orbe di tenebra piena, / tu lontano dagli uomini, / uccello erinni, indovinami il cuore» (Giorno a Capo Soprano, p. 758, vv. 12-15: tre versi strapieni di 'ripellinismi' - l'evocazione raddoppiata, l'avverbio di tempo «presto», il verbo «indovinami» che è fonte di interessanti slittamenti e contrazioni semantiche, l'accoppiata nominale «uccello erinni» dove non è facile individuare quale dei due sostantivi abbia funzione aggettivale - emergenti in una poesia che nel complesso sovrabbonda, piuttosto, di mosse tipicamente quasimodiane);

- «Oh mentitemi, ditemi ch'è vivo» (Suasoria, p. 766, v. 14).

13 La sintassi è certamente un campo entro il quale si costruisce l'idioletto stilistico di questi due raffinati scrittori. Ma il loro stile assurge a inimitabile e consonante marchio di fabbrica nell'ambito lessicale ${ }^{19}$. I fenomeni che indicherò risultano tanto più indicativi in quanto il lessico dell'Amaro miele risulta parecchio più sobrio e meno riccamente inventivo rispetto a quello dei testi in prosa. Infatti, da un recente studio di Gualberto Alvino si ricava che i campioni di neologismi, neoformazioni, forme antiquate, individuati dallo studioso come significativi setacciando tutta l'opera bufaliniana, raramente appartengono alla produzione poetica. È pur vero che, rispetto all'analisi di Alvino, è proponibile qualche integrazione, ma l'assunto di fondo viene confermato. E tuttavia, se già l'esemplificazione che sto per produrre è sufficiente a individuare i contorni dell'ampio dialogo intertestuale tra Bufalino e un prodigioso creatore e manipolatore di lessico quale fu Ripellino, è facile immaginare che, quando si potrà estendere la comparazione alla produzione prosastica, i risultati saranno ancora più eclatanti. Ma procediamo ordinatamente.

14 La lirica ripelliniana, ma anche la sua prosa, è costellata di nomi propri: nomi di persone in carne e ossa, di scrittori e artisti celebri o malnoti, di personaggi storici o del tutto inventati, di personaggi letterari riusati simbolicamente $o$, il più delle volte, grottescamente deformati, sotto forma tanto di alter ego che di identità separate (gli esempi sono innumerevoli: basti qui rinviare ai già citati versi incipitari di La fortezza d'Alvernia 1). Altrettanto accade nella prosa bufaliniana, con punte rilevabili in tutti i romanzi ma anche in un libro come Museo d'ombre, nel quale l'onomastica di paese assurge a dimensione epica, sia pure in minore. Non sorprende che nelle poesie i casi siano molto più rari; eppure non mancano gli echi dell'onomastica siciliana («Compare Cardullo», Qualche canto mafioso 3, p. 746, v. 5) o i nomi evocativi, soprattutto della stagione bellica, come nella lirica Requiem per il nemico ignoto (pp. 723-725): «Adolf Enne Enne» (v. 2), «Rudolf Chissachì» (v. 15), «Gretche, Liselotte» (v. 45), «Hermann Senzanome» (v.60), dove ai tipici nomi maschili evocanti nazionalità tedesca si associano cognomi che significano anonimato, impossibilità d'individuare il milite ignoto, necessità di allargare la tragedia della guerra a un popolo intero, fosse pure il 
nemico. Molto più interessante appare una poesia come Versi per uno spettro (p. 807), nella quale si riflette metalinguisticamente sulla nominazione $-\ll$ No, non ti chiami Erminia, né Claudia, né Rosa, / [...]. // Via di qui, Ics Ypsilon, non ti dirò più niente» (vv. 5 e 15) - per poi approdare al neologismo «Gesùcrista» (v. 13), vertiginoso per l'accentazione e per lo slittamento di genere, che Alvino qualifica come coniazione d'autore, l'unica dell'Amaro miele insieme a un più innocuo «primagosto» (Qualche canto mafioso 1, p. 745, v. 2).

Però, per dire dell'effervescenza onomastica che Bufalino condivide con Ripellino, ai neologismi d'autore bisogna aggiungere le antonomasie ("Questo nel mio bagaglio di romeo", Foglio di viaggio, p. 741, v. 9); la toponimia, che ovviamente è molto ricca per quanto riguarda la Sicilia e segnatamente la natia provincia iblea, ma comprende anche «Tartaro o Sempione» (Stazione notturna, p. 764, v. 4), e un ben significativo "ponte della Bettola», evocante un'atroce rappresaglia nazista (Poscritto, dopo molti anni, p. 811, v. 25); le varianti antiquate come «fantasima» (Per un sogno antelucano, p. 732, v. 6) e i non pochi arcaismi, o altre parole rare e d'uso letterario: non soltanto l'«angue» (Requiem per il nemico ignoto, p. 723, v. 18) segnalato da Alvino, ma anche «indoliti» (Agli amici morti 1, p. 727, v. 8), «postema» (Per un sogno antelucano, p. 732, v. 4), «accordellata nel busto d'alpagio» (Salmo dello scapolo alla fiera, p. 778, v. 6), «druda» (Scioglilingua per piacerle, p. 779, v. 10).

Né si possono dimenticare le citazioni musicali, tipiche dell'intertesto ripelliniano e bufaliniano, come quelle cinematografiche, artistiche, letterarie (meno presenti o del tutto assenti, però, nell'Amaro miele): e siamo al vertice delle passioni musicali di Bufalino, con Mozart nel titolo di una poesia (Eine kleine Nachtmusik, p. 785) e in una citazione della sonata n. 21 per violino K. 304 (abbreviata come K. 304 in Congedi 8, p. 713, v. 13), e con il jazz colto eppure amabile di George e Ira Gershwin evocato dalla menzione di Embraceable you in Cartolina militare (p. 805, v. 11).

Ma il fenomeno lessicale più rimarchevole nel gioco intertestuale che sto provando a ricostruire mi sembra un altro. Mi riferisco a una specificità del lessico ripelliniano ${ }^{20}$ che è l'aggettivo di derivazione nominale accoppiato, e quasi sempre preposto, al sostantivo. Ecco i luoghi più interessanti che ho trovato nell'Amaro miele, con qualche osservazione a margine:

- «sbarrami in viso le streghe pupille» (Parole da lontano, p. 737, v. 5);

- «Allora m'incammino / nel sonnambulo sole» (Bagattella all'antica, p. 760, vv. 5-6);

- «Facile cuore, femmina cuore» (Lamento del facile cuore, p. 763, v. 18: dove tutto è reso più complesso e ritmicamente fascinoso dalla simmetria rafforzata dall'allitterazione, con più d'un sospetto di associazione d'idee tra 'facile' e 'femmina');

- «d'un minuto rancore, d'un bambino rancore» (Poscritto, dopo molti anni, p. 812, v. 35: con effetti analoghi a quelli appena descritti);

- «e la malinconica luna / piace ancora ai miei occhi cervieri» (Ritorno sulla collina, p. 740, vv. 11-12), letteratissimo recupero da Pulci (cfr. Morgante, 22, 254: «Che in campo d'oro portava un cerviere») dove il sostantivo plurale non ha niente a che fare con 'cervo' ma è antico sinonimo di 'lince'. Citazione che conferma l'affezione di Bufalino per la tradizione del poema cavalleresco, non solo nella declinazione popolaresca da cui deriva la sua riscrittura del Guerrin Meschino o in quella 'alta' a cui va riferito il suo conclamato amore per il Boiardo ${ }^{21}$, ma anche in quella seriocomica del Pulci. bufaliniana che, in modo diverso, suoneranno familiari all'orecchio di chi ha confidenza 
con la scrittura di Ripellino. E che, in forza di questa 'familiarità' - insieme sintattica, lessicale, ritmica e fonica - potrebbero configurarsi come vere e proprie citazioni oppure più vaghi omaggi intertestuali dell'anziano scrittore comisano al meno longevo autore di Praga magica.

Inizierei da «[...] fisso a guardare nell'orto / un albero di ciliegio teatralmente morire» (Dedica, dopo molti anni, p. 695, vv. 11-12), affratellante citazione del teatro čechoviano, carissimo a Ripellino, che utilizza l'effetto rafforzante dell'avverbio di modo secondo una strategia testuale che Bufalino aveva già sperimentato per citare Dante nel primo, formidabile capitolo di Diceria dell'untore: «Non restava che procedere un poco, ed ecco, al posto di sempre, purgatorialmente seduti a ridosso l'uno dell'altro, uomini vestiti d'impermeabili bianchi, e si scambiavano frantumi di suono, una poltiglia di sillabe balbe rimasticate in eterno da mascelle senili $»^{22}$.

Si può continuare rammentando l'incipit dell'autoriale Nota alla prima edizione dell'Amaro miele - «Questi versi [...] divenuti, invecchiando, patetici come rullo di pianola o vecchie fotografie» (p.693) - che affratella i due scrittori non soltanto perché raggiunge quel tono a loro carissimo che si potrebbe definire 'patetismo dell'antiquato' ma anche per l'esplicita menzione d'un oggetto elettivamente ripelliniano come la pianola meccanica a rulli. Analoghi echi sono suscitati da altre evocazioni delle giocolerie circensi o da kermesse come mezzi per esorcizzare la sofferenza:

- "Queste parole di un uomo dal cuore debole, / sorta di macchine o giochi per soffrire di meno» (Dedica, dopo molti anni, p. 695, vv. 1-2);

- «Ma dove sei, ora che ottobre verbera / le tende e i cosmorami della sagra, / e nessuno ci chiama / dei giocolieri al suo giallo riverbero» (Cartolina militare, p. 805, vv. 1-4);

- Un po' tutta la lirica Malincuore, il giorno del santo (p. 762), piena di «venditori, / mangiaspade, mangiafuoco» (vv. 2-3) e che si conclude così: «Io non ho fiere più da visitare, / e più m'attempo più voglio morire» (vv. 8-9), dove ogni speranza pare raggelata dal verbo dantesco-petrarchesco.

21 Altre immagini o cadenze tipicamente ripelliniane aggallano qua e là nelle liriche di Bufalino: «l'inverno nel suo mantello d'ussaro» (Dedica, dopo molti anni, p. 695, v. 20); «Passerà sul tuo petto il ferro dei convogli, / e uomini, cantando [...]» (Congedi 2, p. 710, vv. 1-2); «Venire nel tuo povero reame / quest'inverno, senza rumore, / sentendo d'un tratto nel cuore / la morte come una fame» (Congedi 3, p. 711, vv.1-4); "Giunta è la pioggia, la sozza, la losca: / una compagna d'obbrobrio, una mezzana di pena» (Fogli dal diario d'inverno 2, p. 720, vv. 1-2); «[...] Né voglio più sentirla / con unghie mozze sulle tegole sfinirsi; / tentarci col lagno, col lezzo, / a una sorte di guaste sementi; / in un pugno di fradicia terra / riproporci monotona la morte» (ivi, vv. 6-11); «Nei miei occhi si sporca una rosa, / come un albero cadrò. / O Signore, concedimi sull'erba / una morte di cosa» (Compieta, p. 726, vv. 9-12).

Ma se volessimo insistere su quest'esercizio - dell'orecchio, della ragione e anche del cuore - oltre a Lamento del facile cuore (p. 763) da cui si sono trascelti non pochi versi ad esempio, c'è un'altra poesia di Bufalino, A se stesso (p. 765), leopardiana nel titolo ma che scorre tutta su motivi, cadenze e lessemi ripelliniani, talché forse vale la pena di trascriverla, per concludere, per intero:

Un giorno

sarò forse famoso,

sbaglieranno il mio cuore in dieci lingue. 
Ma i silenzi e le urla

allora saranno finiti,

e l'ambulare dispari del sangue.

Acqua nera verrà sul tuo dannato

soliloquio, sul tondo

campiello dove alla cerca t'aggiri,

dio spaventato, carceriere schiavo,

dolcissimo vocabolo di sonno,

ipotalamo insonne.

\section{NOTE}

1. G. Bufalino, Il malpensante. Lunario dell'anno che fu, Milano, Bompiani, 1987, ora in Id., Opere 1981.1988, a cura di M. Corti e F. Caputo, Milano, Bompiani, 1992, p. 1112.

2. È pur vero che lo splendido carteggio tra Bufalino e Angelo Romanò dimostra che, nel caso specifico, l'amicizia nata sotto le armi e la sintonia intellettuale sono indistinguibili: cfr. A. Romanò e G. Bufalino, Carteggio di gioventù (1943-1950), a cura di N. Zago, Valverde, Il Girasole Edizioni, 1994.

3. A. M. Ripellino, La fortezza d'Alvernia e altre poesie, Milano, Rizzoli, 1967 (ora in Id., Poesie prime e ultime, a cura di F. Lenzi e A. Pane, Torino, Aragno, 2006); G. Bufalino, Diceria dell'untore, Palermo, Sellerio, 1981 (ora in Id., Opere 1981.1988, cit.).

4. Tutti presenti presso la Biblioteca comunale di Comiso, quotidianamente frequentata da Bufalino il quale sovente consigliava l'acquisto dei libri al direttore, e in qualche caso anche nella sua biblioteca personale, ora conservata presso la Fondazione Gesualdo Bufalino di Comiso.

5. A. M. Ripellino, Non un giorno ma adesso, Roma, Grafica, 1960 (ora in Id., Poesie prime e ultime, cit.).

6. Per le notizie di carattere biobibliografico cfr. F. Caputo, Cronologia e Note ai testi, in G. Bufalino, Opere 1981.1988, cit.; A. M. Ripellino, Bibliografia, a cura di A. Pane, «eSamizdat», vol. II, 2004, pp. 251-274; A. Pane, Notizia biografica, in A. M. Ripellino, Notizie dal diluvio. Sinfonietta. Lo splendido violino verde, a cura di A. Fo, F. Lenzi, A. Pane e C. Vela, Torino, Einaudi, 2007.

7. Alludo, naturalmente, ad A. M. Ripellino, Letteratura come itinerario nel meraviglioso, Torino, Einaudi, 1968. Su questo volume, mi permetto di rinviare a G. Traina, Materiali per uno studio sul Ripellino saggista, in N. Zago, A. Schininà e G. Traina (a cura di), Angelo Maria Ripellino e altri ulissidi, Atti del convegno di studi (Ragusa, 6-7 aprile 2016), Leonforte, Euno Edizioni, 2017, pp. 59-75.

8. S. Scalia, Una comune sensibilità barocca, in A. M. Ripellino poeta-slavista, fascicolo monografico di «Lunarionuovo», vol. V, $\mathrm{n}^{\circ}$ 21-22, p. 144. Il corsivo è mio.

9. A. M. Ripellino, Il trucco e l'anima. I maestri della regia nel teatro russo del Novecento, Torino, Einaudi, 1965.

10. N. Zago, Per rileggere «Diceria dell'untore», in Id., I sortilegi della parola. Studi su Gesualdo Bufalino, Leonforte-Comiso, Euno Edizioni-Fondazione Gesualdo Bufalino, 2016, p. 72, n. 43.

11. La silloge fu pubblicata nel 1982 da Einaudi e raccoglieva poesie scritte, in gran parte, nel decennio 1944-1954, con qualche altro apporto di epoca successiva. In particolare, di precisa ambientazione sanatoriale è la prima sezione, Annali del malanno, che ospita liriche composte tra il '44, l'anno in cui Bufalino si ammala, e il '47, l'anno in cui guarisce. Il libro ebbe due successive edizioni, nell'89 e nel '96, presso lo stesso editore, con aggiunte di poesie giovanili e degli ultimi 
anni. La presente analisi è condotta però sulla prima edizione, riprodotta in G. Bufalino, Opere 1981.1988, cit., da cui si ricaveranno le citazioni testuali, d'ora in poi indicando nel testo semplicemente titolo della poesia, pagina in cui si trova nella suddetta edizione ed eventualmente i versi a cui ci si riferisce. Non si può dire che questa raccolta sia l'unica di Bufalino, ma è certamente la principale, perché si possono aggiungere due plaquettes - I languorie le furie. Quaderni di scuola (1935-1938), Valverde, Il Girasole edizioni, 1995, e Senilia, Senningerberg, Origine, 1996 -, peraltro parzialmente presenti nell'ultima edizione dell'Amaro miele.

12. A. M. Ripellino, Autunnale barocco, s. l., Guanda, 1977 (ora in Id., Poesie prime e ultime, cit.).

13. Nella maggior parte dei casi, le poesie di Ripellino non hanno titolo ma sono invece numerate, con numeri arabi, suggerendo così un'intenzione poematica che viene poi confermata dalla compattezza e coerenza delle varie raccolte, o delle singole parti di esse.

14. A. M. Ripellino, La fortezza d'Alvernia 5, vv. 10-14, in Id., Poesie prime e ultime, cit., p. 127.

15. Cfr. A. M. Ripellino, Notizie dal diluvio, Torino, Einaudi, 1969, ora in Id., Notizie dal diluvio. Sinfonietta. Lo splendido violino verde, cit.

16. In Notizie dal diluvio. Sinfoniette. Lo splendido violino verde, cit., p. 45, vv. 14-19.

17. La fortezza d'Alvernia 1, in Poesie prime e ultime, cit., p. 123, vv. 1-3.

18. Si pensi soprattutto alle poesie di Notizie dal diluvio, indelebilmente segnate dall'ansia per la sorte degli amici intellettuali e artisti praghesi, sodali di Anton Dubček e, come lui, travolti dalla repressione sovietica del luglio '68. Su questa raccolta, ma anche sull'influsso di Čechov, cfr. U. Brunetti, Dalle «Tre sorelle» alla Primavera di Praga: la poesia $n .76$ di «Notizie dal diluvio», in Angelo Maria Ripellino e altri ulissidi, cit., pp. 43-58.

19. Sulle specificità lessicali dei due scrittori cfr. G. Dierna, Rileggere Ripellino, in A. M. Ripellino poeta-slavista, cit., pp. 71-79, e G. Alvino, Effetto Ikebana, in N. Zago e G. Traina (a cura di), Il miglior fabbro. Bufalino fra tradizione e sperimentazione, Leonforte, Euno Edizioni, 2014, pp. 19-43.

20. Come al solito, basterà un esempio: «sparviera tristezza», da La fortezza d'Alvernia 7, v. 8, in Poesie prime e ultime, cit., p. 129.

21. Cfr. G. Bufalino, Il Guerrin Meschino. Frammento di un'opra dei pupi, Milano, Bompiani, 1993, ora in Id., Opere/2 1989.1996, a cura di F. Caputo, Milano, Bompiani, 2007; Matteo Maria Boiardo, introduzione e scelta di G. Bufalino, Roma, Istituto Poligrafico e Zecca dello Stato, 1995.

22. G. Bufalino, Diceria dell'untore, cit., p. 10. A proposito di questa modalità citazionistica, e di tutto il capitolo incipitario, vorrei rinviare al mio studio La vertigine di un incipit e l'espiazione dei sogni, in G. Traina, «Lafelicità esiste, ne ho sentito parlare». Gesualdo Bufalino narratore, Cuneo, Nerosubianco, 2012, pp. 11-15.

\section{RIASSUNTI}

L'articolo offre i primi risultati di un confronto intertestuale tra le poesie di Gesualdo Bufalino (L'amaro miele, 1982) e le opere di Angelo Maria Ripellino: l'analisi è condotta sulla metrica, la sintassi, il lessico.

L'article propose les premiers résultats d'une comparaison intertextuelle entre les poèmes de Gesualdo Bufalino (L'amaro miele, 1982) et les œuvres d'Angelo Maria Ripellino : l'analyse porte sur la métrique, la syntaxe, le lexique et les thèmes. 
The article offers the first results of an intertextual comparison between the poems of Gesualdo Bufalino (L'amaro miele, 1982) and the works of Angelo Maria Ripellino: the analysis concerns metrics, syntax, vocabulary and themes.

\section{INDICE}

Parole chiave : intertestualità, Bufalino, Ripellino Keywords : intertextuality, Bufalino, Ripellino

Mots-clés : intertextualité, Bufalino, Ripellino

\section{AUTORE}

GIUSEPPE TRAINA

Università di Catana 